\title{
Electron holography observation of magnetic nanostructures formed by electron beam induced chemical vapor deposition with $\mathrm{Fe}(\mathrm{CO})_{5}$
}

\author{
M. Takeguchi*, M. Shimojo*,**, R. Che*, and K. Furuya* \\ * High voltage electron microscopy station, National Institute for Materials Science, 3-13 Sakura, \\ Tsukuba, 305-0003, Japan \\ ** Precision and Intelligence Laboratory, Tokyo Institute of Technology, 4259 Nagatsuta-cho, \\ Midori-ku, Yokohama, 226-8503, Japan.
}

Nanometer-sized ferromagnetic structures have attracted much attention because of their interesting properties and potential of practical application to advanced submicron magnetic devices. An understanding of the switching behavior of the magnetic nanostructures has been significant issue in point of view of application to high density magnetic recording and magnetic random access memory. Properties of the magnetic nanostructures strongly depend on their shape and size [1]. Therefore, recently, much effort to fabricate the magnetic nanostructures with the desired shape at the desired position has been made [2]. Electron beam induced chemical vapor deposition (EBICVD) is one of the most prospective methods for size and position controllable fabrication [3]. We have found that iron-based nanostructures could be formed by this method with $\mathrm{Fe}(\mathrm{CO})_{5} \mathrm{gas}$ and a post-heating treatment could transform them into single crystal alpha-iron phase without shape change. In the present work, their magnetic flux distribution was observed by electron holography.

A $30 \mathrm{kV}$ FEG-SEM, JSM-7800F-UHV, was employed to carry out EBICVD experiments. $\mathrm{Fe}(\mathrm{CO})_{5}$ gas was introduced into the specimen chamber through a nozzle. The partial pressure of the introduced gas was controlled to be about $7 \times 10^{-5} \mathrm{~Pa}$ using a valuable leak valve. Nanometer-sized dots, rods and square frames were fabricated on a silicon film at room temperature. An electron beam current was about $0.8 \mathrm{nA}$ with a beam diameter of $4 \mathrm{~nm}$. The heating treatment was done in a UHV chamber. The residual magnetic flux density $B_{r}$ of the as-formed and alpha-iron nanorods was quantitatively measured after magnetization in a field emission transmission electron microscope JEM-3000F equipped with an electron biprism.

Figure 1 shows a transmission electron microscopy (TEM) image of variously-shaped self-standing nanostructures formed on a thin crystalline silicon substrate edge at room temperature. The selfstanding nanostructures were fabricated by moving the beam from the silicon film edge to space at a speed of 2-3 m/s. Their linewitdh was 30-50nm.

Figures $2 \mathrm{a}$ and $2 \mathrm{~b}$ show TEM images of the self-standing nanostructures on a silicon film edge before and after a post-deposition heating at about $600{ }^{\circ} \mathrm{C}$ for 2 hours, respectively. Figure 2c is a dark field TEM image of the nanostructure labeled by $B$ in Fig. $2 \mathrm{~b}$. It should be noted that the shape of the nanostructures was almost maintained after heating. It was confirmed that the heated nanostructure was composed of single crystal alpha iron by electron diffraction and dark-field TEM.

Figure $3 \mathrm{a}$ and $3 \mathrm{~b}$ show electron hologram and reconstructed interference micrograph of the asformed nano-rod with a ring head indicated by an arrow in Fig. 1. Phase is amplified by 4 times. The phase difference between both the sides of the nanorod was about $4.3 \mathrm{rad}$, which corresponds to the 
magnetic flux of $2.31 \times 10^{-15} \mathrm{~Wb}$, and then the residual magnetic flux density $B_{r}$ was calculated to be about $0.61 \mathrm{~T}$. In case of the heated nanostructure shown in Fig. $2 \mathrm{c}$, the $B_{r}$ value was about $0.45 \mathrm{~T}$. It was found that the $B_{r}$ value of the alpha-iron nanorod is smaller than that of the as-formed nanorod.

[1] D. J. Smith et al., J. Apply. Phys. 87 (2000) 7400.

[2] S. P. Li et al., J. Magn. Magn. Mater. 241 (2002) 447.

[3] H. W. P. Koops et al., Jpn. J. Appl. Phys. Part1, 33 (1994) 7099.

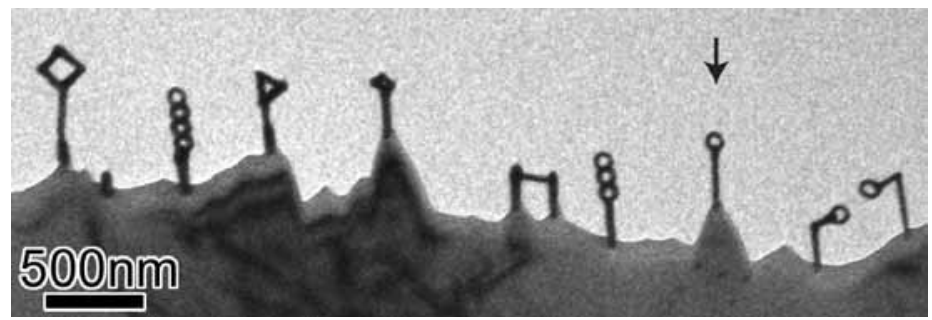

FIG. 1 TEM image of the desired-shape self-standing nanostructures on a thin silicon substrate edge formed by $\mathrm{EBICVD}$ with $\mathrm{Fe}(\mathrm{CO}) 5$ at room temperature.
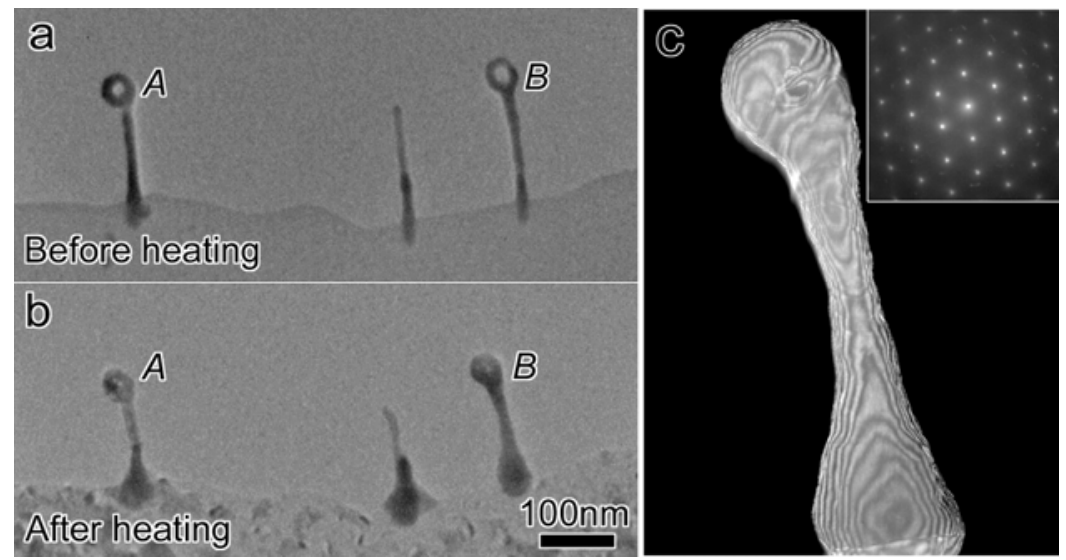

FIG. 2 TEM images of the free standing nanostructures on a silicon substrate before (a) and after (b) a heat treatment at about $600 \mathrm{oC}$ for 2 hours. (c) Dark field TEM image of the nanostructure labeled by $B$ in (b), in where diffraction pattern is inserted.

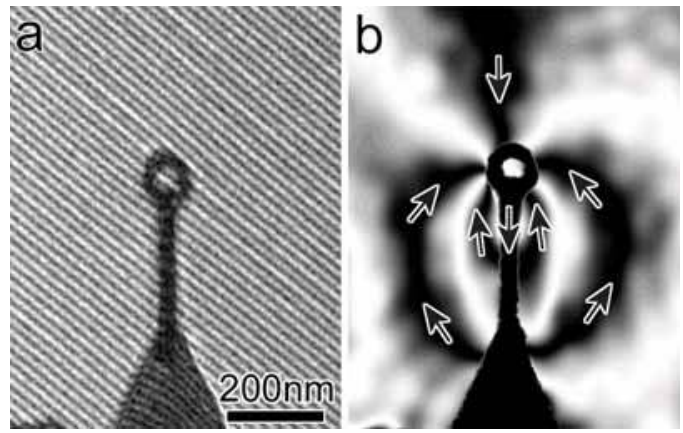

FIG.3 (a) Electron hologram and (b) reconstructed interference micrograph of the as-formed nanorod indicated by an arrow in Fig. 1. Phase is amplified by 4 times. 